\title{
NUMERICAL SIMULATION OF HYSTERETIC LIVE LOAD EFFECT IN A SOIL-STEEL BRIDGE
}

\author{
MACIEJ SOBÓTKA \\ Institute of Geotechnics and Hydrotechnics, Wrocław University of Technology, \\ Wybrzeże Wyspiańskiego 27, 50-370 Wrocław, Poland, e-mail: maciej.sobotka@pwr.wroc.pl
}

\begin{abstract}
The paper presents numerical simulation of hysteretic live load effect in a soil-steel bridge. The effect was originally identified experimentally by Machelski [1], [2]. The truck was crossing the bridge one way and the other in the full-scale test performed. At the same time, displacements and stress in the shell were measured. The major conclusion from the research was that the measured quantities formed hysteretic loops. A numerical simulation of that effect is addressed in the present work. The analysis was performed using Flac finite difference code. The methodology of solving the mechanical problems implemented in Flac enables us to solve the problem concerning a sequence of load and non-linear mechanical behaviour of the structure. The numerical model incorporates linear elastic constitutive relations for the soil backfill, for the steel shell and the sheet piles, being a flexible substructure for the shell. Contact zone between the shell and the soil backfill is assumed to reflect elastic-plastic constitutive model. Maximum shear stress in contact zone is limited by the Coulomb condition. The plastic flow rule is described by dilation angle $\psi=0$. The obtained results of numerical analysis are in fair agreement with the experimental evidence. The primary finding from the performed simulation is that the slip in the interface can be considered an explanation of the hysteresis occurrence in the charts of displacement and stress in the shell.
\end{abstract}

Key words: soil-steel structure, flexible steel culvert, live load effect, hysteresis

\section{INTRODUCTION}

In contrast to rigid arched structures, the flexible ones, constructed typically with corrugated steel plates, are characterised by advantageous effect of interaction between the shell and the soil backfill. The backfill as well as the pavement are considered to be the structural elements of the bridge [3]. The aforementioned benefit, relatively low costs and short time of construction contribute to increasing interest in the technology from the practical as well as theoretical point of view [4]. However, the technology has drawbacks, too. Design and description of the mechanical behaviour of those structures is difficult. The behaviour of corrugated buried structures is significantly non-linear [1], [2], [4]-[8]. That appears to be an obvious fact insofar as one of the materials used in construction is a soil, which is non-linear in nature. Non-linear behaviour of soil-steel bridges exhibits in a few effects, inter alia, changes in stress level under long term load [1], [9], [10] and hysteretic effect under live load. The latter was identified experimentally by Machelski [1], [2]. In the tests performed the load was produced by a truck slowly moving through the bridge in one direction and then back. The displacement and stress in the shell were measured during the entire test. The curves corresponding to the mechanical quantities formed hysteresis loops. A numerical simulation of that hysteretic effect is addressed in the paper.

A considerable number of papers reporting the numerical investigations on the behaviour of flexible structures have been published [1]-[3], [5]-[15]. However, the only attempt to simulate numerically hysteretic effect, according to the author's knowledge, was reported in [15]. Nevertheless, the compatibility of the results obtained with the experimental evidence was not satisfactory. The contribution of the present work to solving the problem of hysteresis is considerable. In comparison to results reported in [15], the results presented in this paper show much better compatibility with the results of experimental full-scale test [2].

The paper is organised in the following way. The next section gives a description of the bridge tested by Machelski [2]. Subsequently, selected measurement results obtained are cited [2]. Succeeding section lays out the computational model. Then, the results of the analysis performed are presented and discussed. The summary and conclusions end the paper. 


\section{SOIL-STEEL BRIDGE STRUCTURE UNDER CONSIDERATION}

The bridge considered is a single span flexible structure on the DK 8 route near Niemcza town in Lower Silesia Region, Poland. A longitudinal crosssection of the structure is presented in Fig. 1. measurements taken at point 12 will be referred to only. This point corresponded to the centre of the shell in circumferential direction while in the transverse direction the point 12 was located under the wheel trace. The measurements were taken for a number of different positions of the truck in the longitudinal direction. Initial position of the truck at the beginning of the test is presented in Fig. 2.

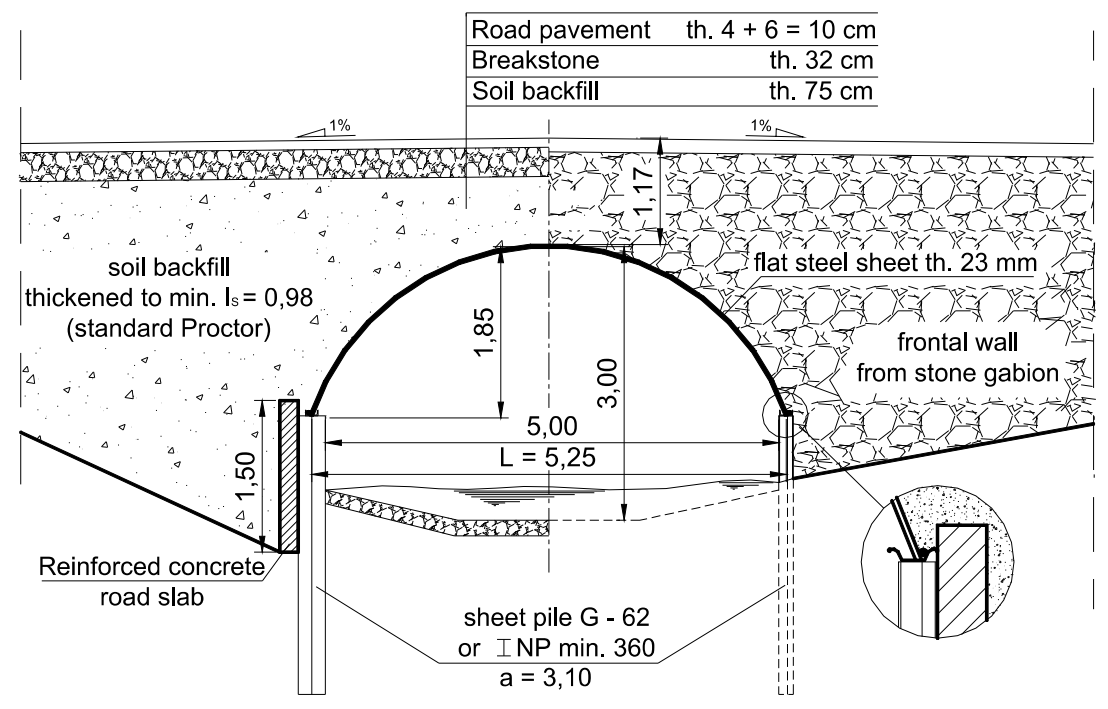

Fig. 1. The longitudinal cross-section of the bridge under consideration [2]

The span length of the bridge is equal to $5.25 \mathrm{~m}$. The shell is made of plain steel sheets $23.0 \mathrm{~mm}$ thick. The shell is founded on steel sheet piles G62 or INP 360 , spaced $3.10 \mathrm{~m}$ and topped with steel girt. The backfill is made of coarse soil compacted to $98 \%$ of SMDD (maximum dry density with respect to the Standard Proctor compaction test). The minimum height of the backfill layer over the shell is $0.75 \mathrm{~m}$. On the backfill layer there is a layer of breakstone, $0.32 \mathrm{~m}$ thick. The road pavement, $0.1 \mathrm{~m}$ thick, forms the top layer. The frontal vertical wall is constructed from stone gabion.

\section{EXPERIMENTAL EVIDENCE OF LIVE LOAD EFFECT}

The research the results of which are reported in [2] consisted in measurements of the displacements and stress in the shell due to the traffic load. The load was produced by Tatra truck moving through the bridge in one direction and the other. The measurements were taken at 10 reference points located on the bottom surface of the shell. In the present work, in order to clarify presentation of simulation results, the
The truck reference location was described by the distance $x_{p}$ between the middle truck axle and the bridge central axis. The reference positions were also numbered. The position reference points were spaced at $\Delta x_{i}=0.675 \mathrm{~m}$, i.e., half of the distance between the second and the third truck axles. Position $i=0$ corresponded to the central setting of the middle truck axle, i.e., $x_{p}=0$. The initial location $i=7$ corresponded to the distance $x_{p}=4.725 \mathrm{~m}$. The forces transferred by the axles to the structure were $P_{1}=54.0 \mathrm{kN}, P_{2}=$ $129.0 \mathrm{kN}, P_{3}=102.0 \mathrm{kN}$. Force $P_{1}$ corresponded to the front axle, $P_{2}$ and $P_{3}$ to the middle and the rear one, respectively.

The test covered two passes through the bridge. In the first one, the truck approached the bridge on reverse. After taking the measurements for the initial position of the truck it was moved slowly to another reference position. Then, the values of the stress and displacements were measured in the new position, etc. After the truck reached the position $i=-3 \quad\left(x_{p}=\right.$ $-2.025 \mathrm{~m}$ ), it was driven back through the bridge without turning back. Thus, the truck, set ahead in the direction of $x$-axis during the entire test, was in all of the reference locations (except $i=-3$ ) two times.

The results of measurements taken by Machelski [2] indicate that the curves corresponding to the 


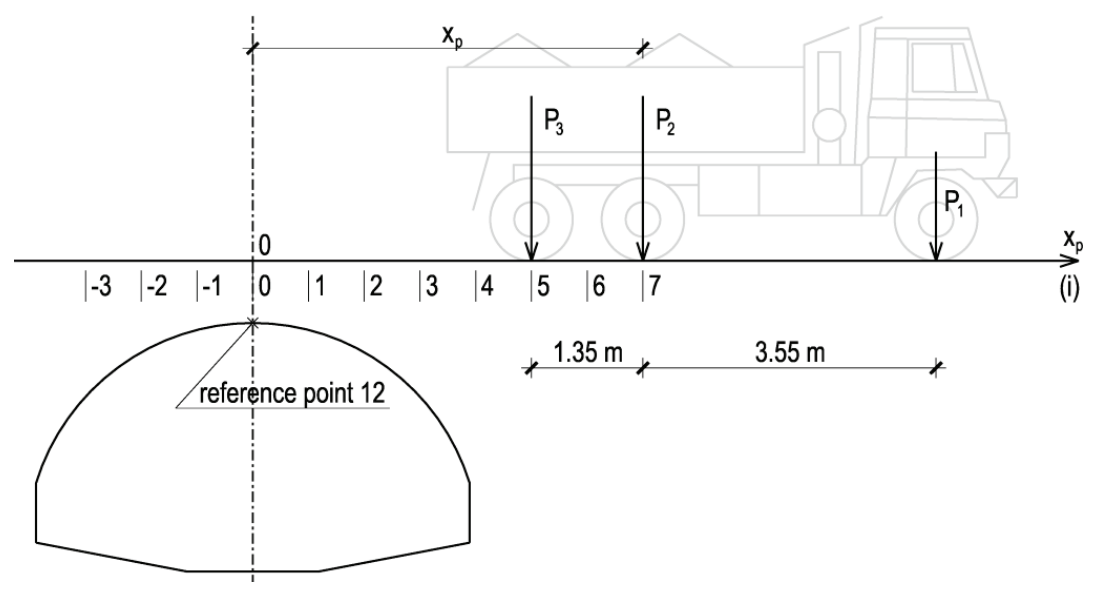

Fig. 2. Scheme of the in-situ test; truck in the initial position

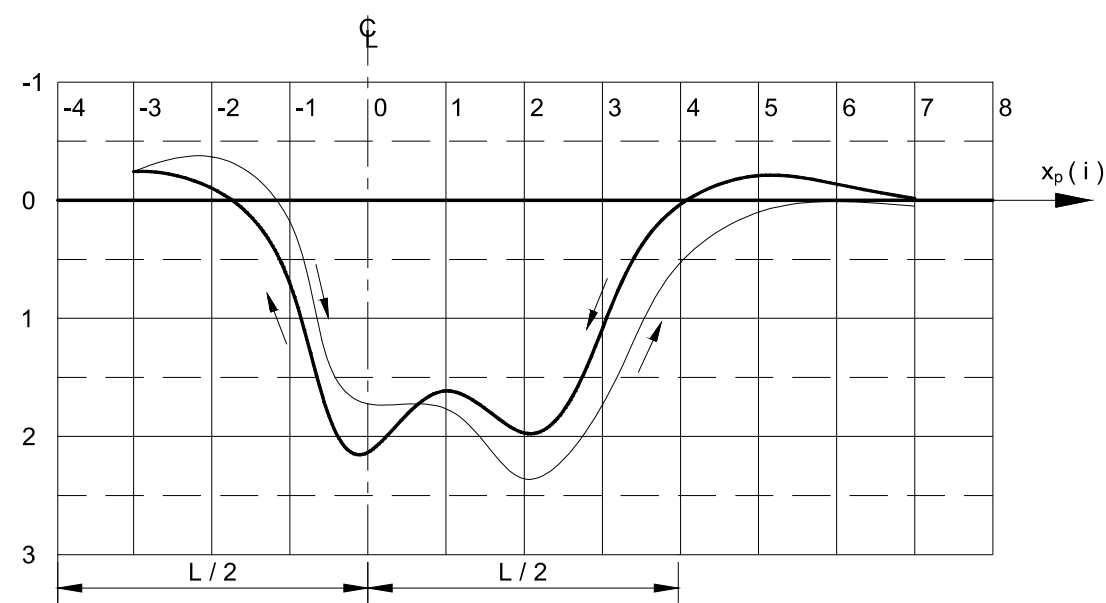

$w \bigvee[\mathrm{mm}]$

Fig. 3. Chart of the vertical displacement $w$ at the measurement point 12 [2]

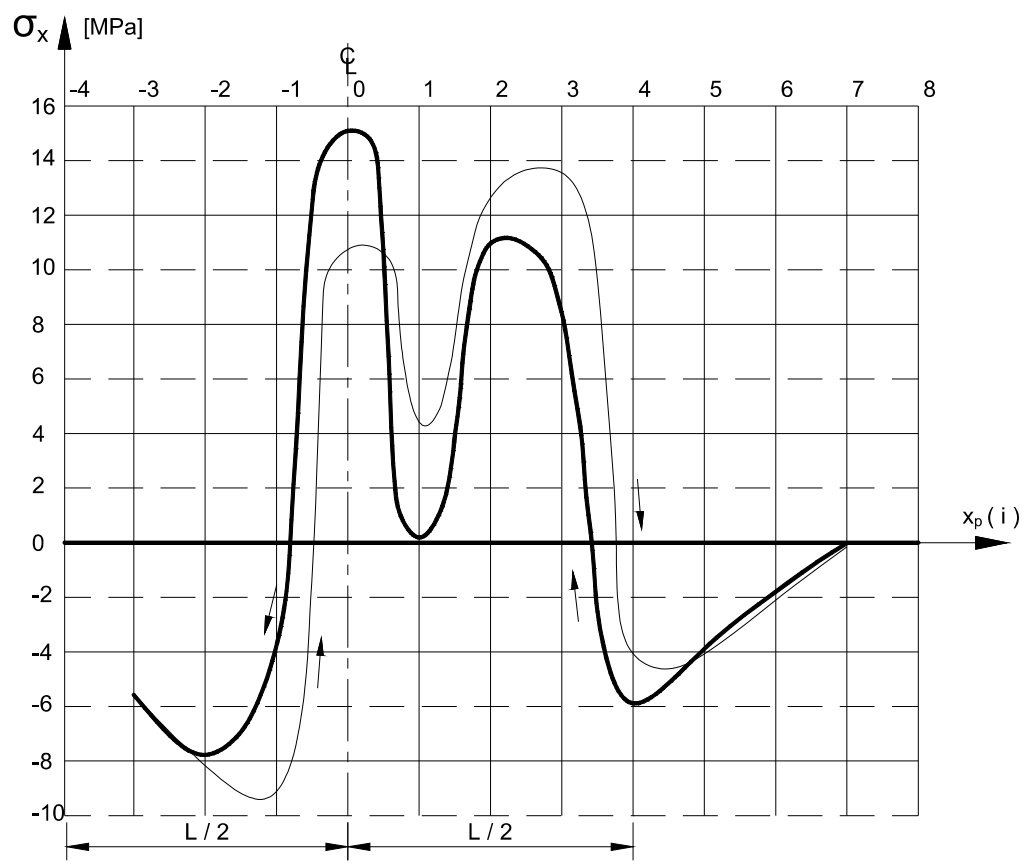

Fig. 4. Chart of the stress $\sigma_{x}$ at the measurement point 12 [2] 
displacements and stress form hysteresis loops. The curves for vertical displacement $w$ of the point 12 as well as the normal stress in circumferential direction $\sigma_{x}$ at the same point are shown in Figs. 3 and 4.

The original test was conducted in two construction phases, i.e., with and without the pavement. The simulations presented in this work will reflect the case without pavement only.

\section{FORMULATION OF COMPUTATIONAL MODEL}

The test described above was simulated with the use of Itasca Flac 7.0 software [16]. Flac is the commercial code using explicit formulation of finite difference method. The full dynamic equations of motion and automated, so called local non-viscous damping are used to provide non-inertial solutions, i.e., static ones or resulting in physically stable plastic flow [16]. Thus, a plastic behaviour of the structure, construction phases or load sequences can be simulated. The above-mentioned properties of that software were the reason of choosing Flac to solve the problem.

The finite difference grid reflects the geometry of the bridge near Niemcza. Structural beam elements create a model of the steel shell and sheet piles. The shell is attached to the soil medium through the so called interface being a model of the contact zone.
The grid as well as the boundary conditions (for initial truck position $i=7$ ) is shown in Fig. 5.

The model consists of 2954 finite difference zones, 92 finite elements of the beam type, and 134 interface elements. The assumptions and material parameters taken in the analysis are given below. Plain strain analysis was assumed. The calculation was performed in large strain mode. Constitutive relations adopted for soil medium were linear elastic with the material parameters as follows: Young's modulus $E=150 \mathrm{MPa}$, Poisson's ratio $v=0.25$, density $\rho=$ $1.9 \mathrm{t} / \mathrm{m}^{3}$. Those values correspond to the parameters of coarse sand in dense state of compaction (density index $I_{\mathrm{D}}=0.8$ ) according to the Polish standard PN-81/B 03020 [17]. The beam elements of the shell were linear elastic. Their geometrical and material parameters were as follows: shell thickness $t_{s}=$ $23 \mathrm{~mm}$, Young's modulus $E_{s}=205 \mathrm{GPa}$ and density $\rho_{s}=7.86 \mathrm{t} / \mathrm{m}^{3}$. It is assumed that the shell is continuous in the out of plain $z$ direction. On the contrary, the steel piles are the reinforcement spaced at $a_{s}=3.1 \mathrm{~m}$. Use of sheet piles G62 was assumed. The cross-section of the sheet piles has the following shape properties: area $A=$ $78.9 \mathrm{~cm}^{2}$, moment of inertia $I=2950 \mathrm{~cm}^{4}$, longitudinal density $\rho=0.062 \mathrm{t} / \mathrm{m}$. Relevant calculation parameters (per $1.0 \mathrm{~m}$ along $z$-axis) result from the abovementioned values divided by $a_{s}$. The parameters were taken as follows: $A_{L}=20.7 \mathrm{~cm}^{2} / \mathrm{m}, I_{L}=983.3 \mathrm{~cm}^{4} / \mathrm{m}$, $\rho_{L}=0.0263 \mathrm{t} / \mathrm{m} / \mathrm{m}$. The Coulomb shear strength criterion limits the shear force within the interface ele-

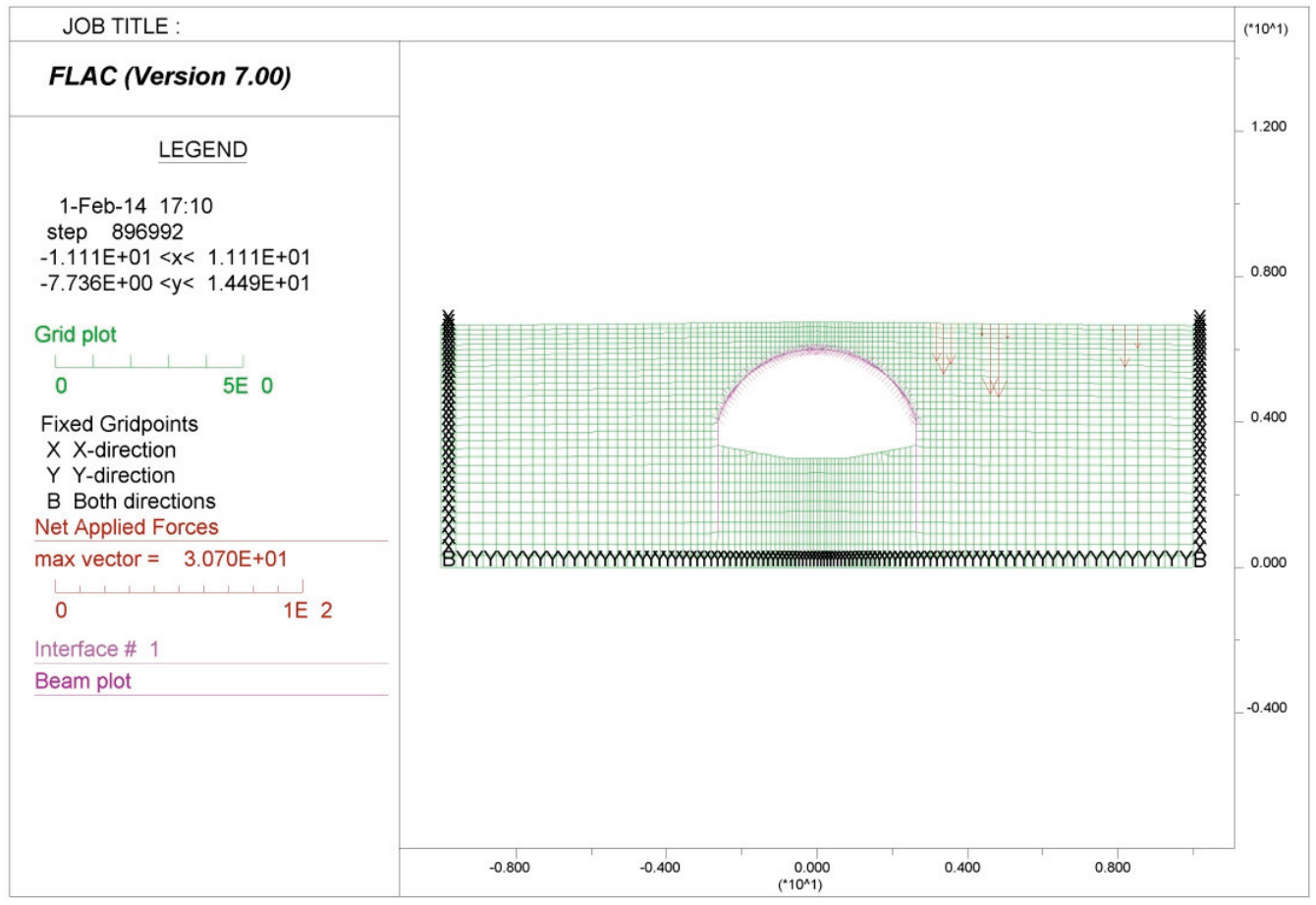

Fig. 5. Computational model of the problem 
ments. The parameters of contact zone are given below: angle of friction $\delta=22 \approx 2 / 3 \phi$, where $\phi=34^{\circ}$, denotes internal friction of backfill soil, i.e., dense coarse sand. The value of adhesion $a=0 \mathrm{kPa}$. Dilation angle was adopted as $\psi=0^{\circ}$. Elastic deformation moduli for interface elements were determined in accordance with Flac Users' Manual [16] as

$$
k_{s}=k_{n}=10 \cdot \frac{K+\frac{4}{3} G}{\Delta z_{\text {min }}}
$$

where $k_{s}, k_{n}$ denote shear and normal stiffness of interface elements; $K$ and $G$ are the parameters of material adjacent to the interface, i.e., bulk and shear modulus, respectively; $\Delta z_{\min }$ corresponds to minimum adjacent zone side length.

\section{RESULTS AND THEIR DISCUSSION}

The results - charts of vertical displacement $w$ and the stress $\sigma_{x}$ at the top point of the shell are presented in Figs. 7 and 8. The black lines in the charts reflect numerical simulation of the original experiment [2], whereas the remaining lines, drawn in grey, reflect subsequent 8 passes (4 in each direction). Hereby, there are results of 10 runs presented in Figs. 7 and 8.

It must be emphasized that the results presented in the charts reflect the excessive values of the displacement $w$ and stress $\sigma_{x}$. In other words, the mechanical state at the beginning of the test stands for the refer-

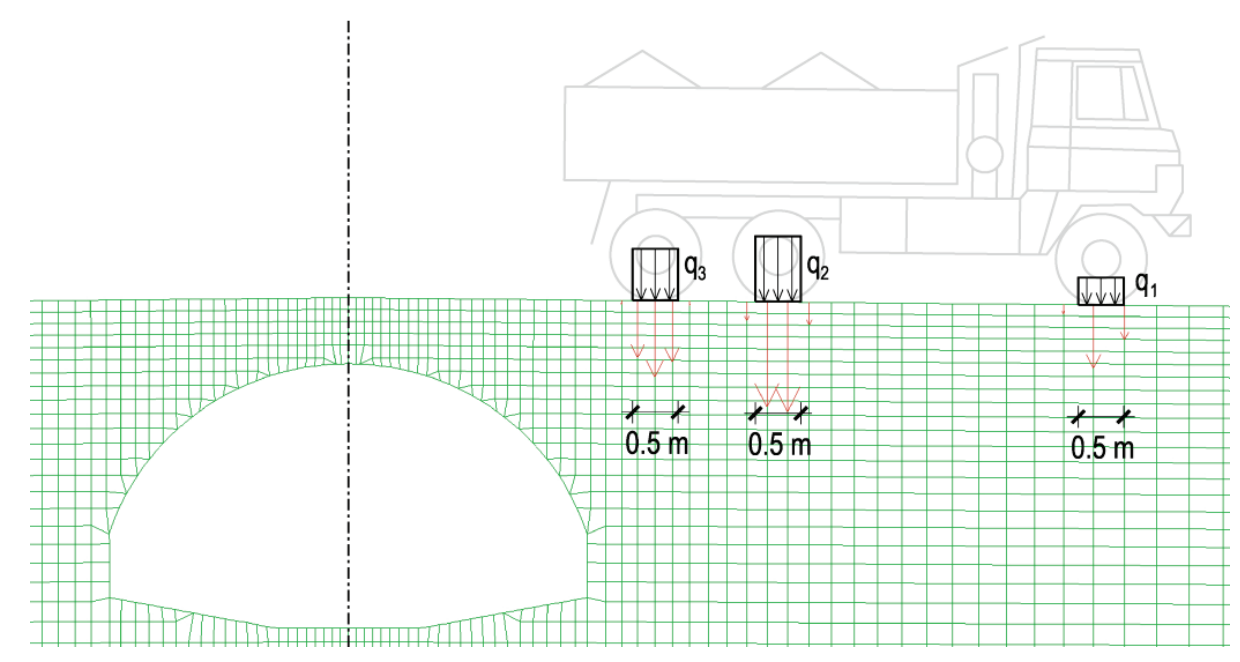

Fig. 6. Scheme of applied traffic load

First stage of the analysis incorporated bringing the model to the state of stress under dead weight load. Then the load produced by the truck was applied. It was assumed that the forces were distributed on the track width in the transverse direction and on a $0.5 \mathrm{~m}$ distance along the bridge. The calculated values of distributed loads were $q_{1}=60 \mathrm{kN} / \mathrm{m}, q_{2}=143.3 \mathrm{kN} / \mathrm{m}$, $q_{3}=113.3 \mathrm{kN} / \mathrm{m}$. Due to the numerical method utilised, the loads were recalculated as equivalent system of forces in the nodes. The scheme of the applied load for position $i=7$ of a truck is presented in Fig. 6 .

For each position, starting from the initial one $(i=7)$, the problem was solved and the outcomes were recorded. Then the subsequent position of the load was set, etc. The distance between subsequent positions was $0.0675 \mathrm{~m}$, i.e., one tenth of the distance between reference points in the original experiment. The simulation covered 10 passes. The obtained results are described in the next section. ence state. Note that the charts start from zero values in the experimental results, too. The calculated values of stress $\sigma_{x}$ result from the equation

$$
\sigma_{x}=\frac{\left(N-N_{0}\right)}{A}+\frac{\left(M-M_{0}\right)}{I} \cdot \frac{h}{2}
$$

where $M, N$ denote actual internal forces at the beam node at the top of the shell, static bending moment and axial force, respectively. $M_{0}=-0.08375 \mathrm{kNm}$ and $N_{0}=$ $-74.94 \mathrm{kN}$ are the reference internal forces calculated for the load case corresponding to the beginning of the test, i.e., dead weight load and the truck in position $i=7$.

The outcomes are in fair agreement with the in situ test result. The maximum value of displacement is almost equal to the one obtained by measurements [2]. The values of the stress $\sigma_{x}$ are underestimated but the course of the chart is in good agreement with the experimental one. It can be easily seen that the values of 


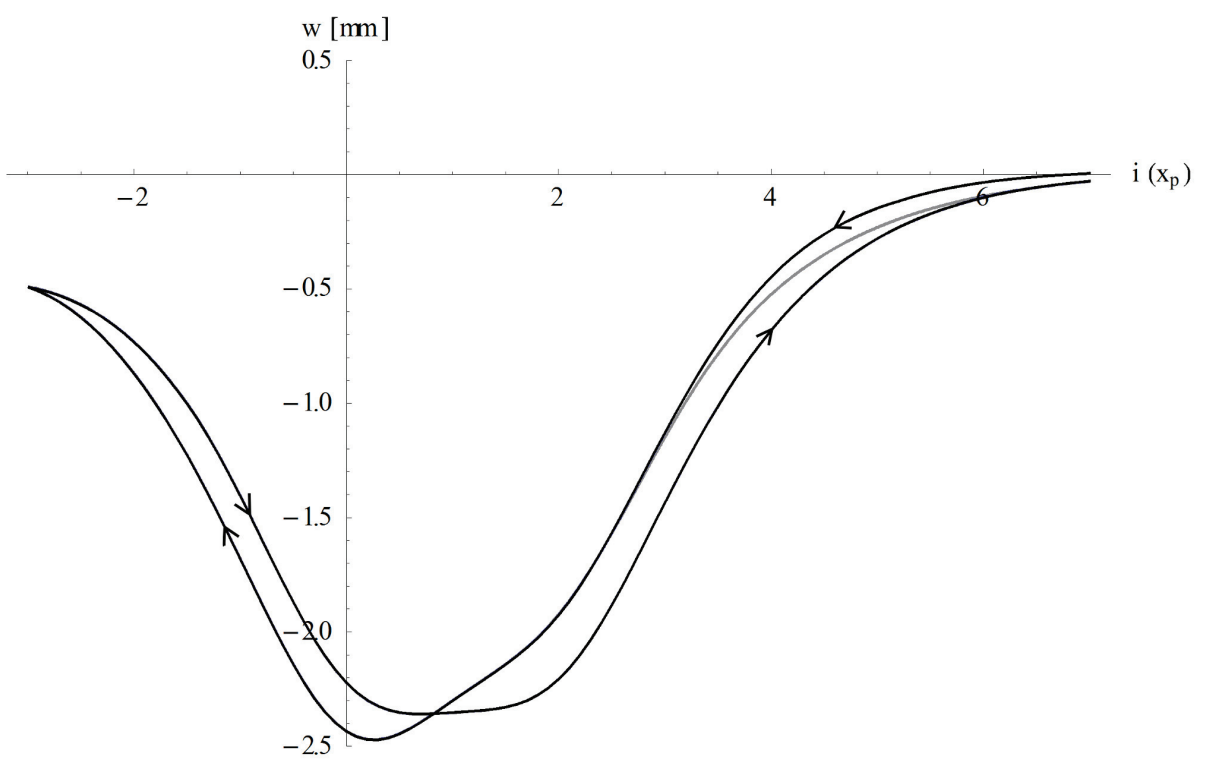

Fig. 7. Chart of vertical displacement $w$ obtained numerically

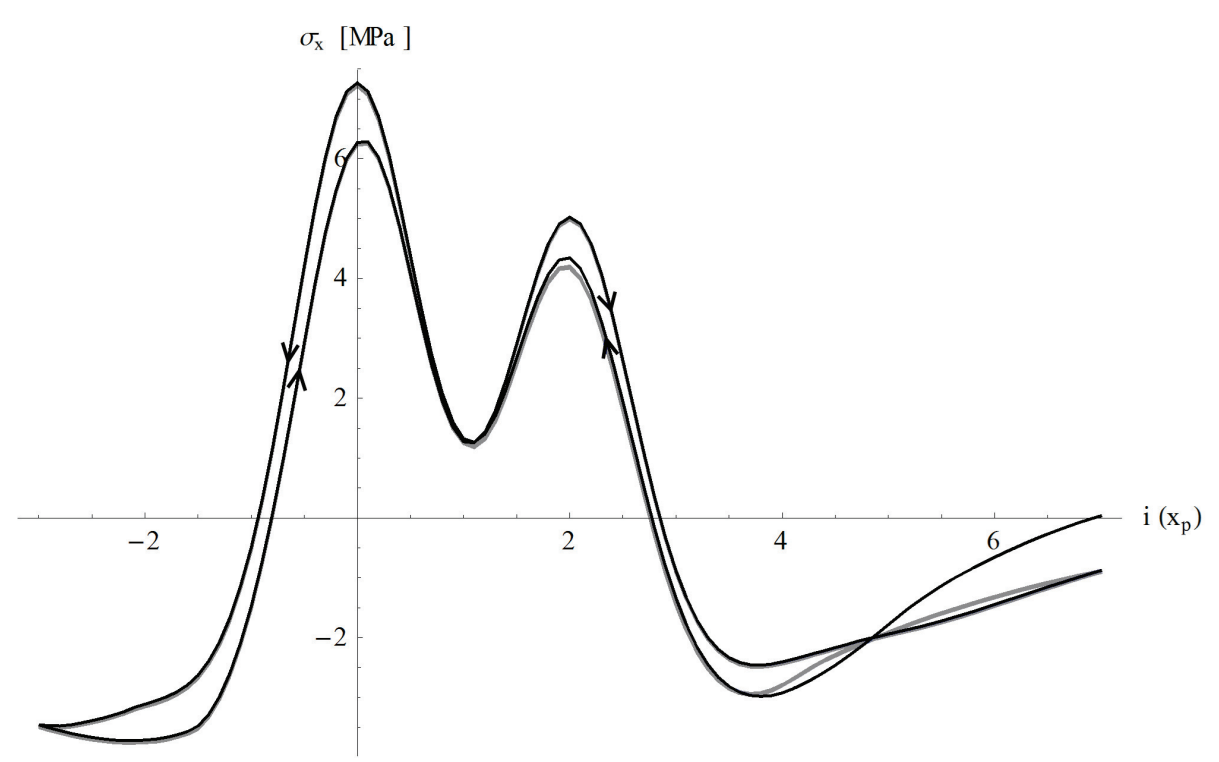

Fig. 8. Chart of stress $\sigma_{x}$ obtained numerically

displacement $w$ as well as peak values of tensile stress $\sigma_{x}$ increase with the pass of the subsequent truck axle for both directions of the truck movement. Note that for linear elastic case (not presented in the paper) hysteresis is not formed, in particular, the peak values associated with a chosen truck axle remain at the same level regardless of the direction of the truck movement. In addition, the locations of extreme values are shifted in the direction of movement for consecutive truck passes. Concluding, the results obtained numerically exhibit the same tendency as those identified experimentally. The value of displacement and extreme value of stress increase with the consecutive axle passes. In addition, the curves are shifted in the direction of the truck movement. Particularly, the peaks associated with consecutive truck axles are shifted as well.

The lines corresponding to the successive runs overlap, except for the first run. This means that the deformation of the shell and relative tangential displacement of the backfill and shell in the contact zone are reversible and repeatable, at least within the framework of the assumed model. For each run, a portion of the energy is dissipated by the slip of the soil on the shell. Another part of energy is accumulated as the elastic energy of the shell deformation. The deformation is locally retained by the friction forces in the soil-steel contact zone. The accumulated 
energy is then released during the run of the truck in the opposite direction.

\section{SUMMARY AND CONCLUSIONS}

The hysteretic effect of live load in the soil-steel bridge was simulated numerically. Constitutive model for backfill medium as well as for the shell and the sheet piles was linear elastic. The behaviour of contact zone was described in terms of Coulomb condition. Plastic slip was governed by non-associated plastic flow rule with the dilation angle value set to $\psi=0$.

The construction phases were not taken into account within the analysis. Accounting for them and, hence, the positive effect of shell pre-stressing [12], [18], [19], would result in obtaining more accurate results. Likewise, taking into account more sophisticated constitutive model for soil backfill, incorporating compaction effects, might improve the quality of the outcomes [12]. Nonetheless, the results presented in the paper are in fair agreement with the experimental evidence and stand for the reliable explanation of hysteretic live load effect.

\section{REFERENCES}

[1] MACHELSKI C., Modelling of soil-steel bridge structures, (in Polish), DWE, Wrocław, 2008.

[2] Machelski C., Antoniszyn G., Michalski B., Live load effects on a soil-steel bridge founded on elastic supports, Studia Geotechnica et Mechanica, 2006, 28, 2-4, 65-82.

[3] ANTONISZYN G., Is the road pavement structural element of the bridge?, Geoengineering: roads, bridges, tunnels, (in Polish), 2009, 3, 76-79.

[4] JANUSZ L., MADAJ A., Engineering structures made form corrugated plates, (in Polish), WKiL, Warsaw, 2007.
[5] Elshimi T.M., Three-dimensional Nonlinear Analysis of Deep-corrugated Steel Culverts, 2011.

[6] Katona M.G., A simple contact-friction interface element with applications to buried culverts, International Journal for Numerical and Analytical Methods in Geomechanics, 1983, 7, 371-384.

[7] Lougheed A., Limit States Testing of a Buried Deep-Corrugated Large-Span Box Culvert, 2009.

[8] MacDonald L., Numerical modelling of vehicle loads on buried orthotropic steel shell structures, Diss., 2010.

[9] MaChELSKI C., ANTONISZYN G., Influence of live loads on the soil-steel bridges, Studia Geotechnica et Mechanica, 2004, 26, 3-4, 91-119.

[10] Madaj A., Sturzbecher K., Changes in stress level in a corrugated steel structure under long-term loads, Archives of Institute of Civil Engineering. Poznan University of Technology, 2012, 175-184.

[11] El-SAwY K.M., Three-dimensional modeling of soil-steel culverts under the effect of truckloads, Thin-Walled Structures, 2003, 41, 8, 747-768.

[12] KorUsiewicz L., KunECKI B., Behaviour of the steel boxtype culvert during backfilling, Archives of Civil and Mechanical Engineering, 2011, 11, 3, 637-650.

[13] KUNECKI B., Full-scale test of corrugated steel culvert and FEM analysis with various static systems, Studia Geotechnica et Mechanica, 2006, 28, 2-4, 5-19.

[14] KUNECKI B., KUBICA E., Full-scale laboratory tests and FEM analysis of corrugated steel culverts under standardized railway load, Archives of Civil and Mechanical Engineering, 2004, 4, 4, 41-53.

[15] Machelski C., Marcinowski ., Numerical modelling of moving load effect in a soil-steel bridge, Archives of Institute of Civil Engineering, Poznań University of Technology, 2007, 155-165.

[16] FLAC. Fast Lagrangian Analysis of Continua. User's Guide. Itasca Consultig Group Inc. Minneapolis, 2001.

[17] PN-81/B-03020 - Building soils. Foundation bases. Static calculation and design" (in Polish).

[18] MAChelsKi C., Deformation of steel shells in soil-steel structures during backfilling, Geoengineering: roads, bridges, tunnels, (in Polish), 2010, 6, 24-30.

[19] MiChALSKI J.B., MiChALSKI B., Characteristics of prestressing in soil-steel structures, (in Polish) Archives of Institute of Civil Engineering. Poznań University of Technology, 2010, 8, 215-244. 